\title{
Sentinel node biopsy in breast cancer patients undergoing induction chemotherapy should be performed prior to chemotherapy
}

\author{
Janusz Piekarski
}

Presently, preoperative chemotherapy is administered ever more frequently in breast cancer patients. It is administered in both non-resectable and resectable patients, i.e. those in whose case it is possible to undertake surgical treatment without the use of chemotherapy. Sentinel node biopsy may only be performed in patients classified as cNO. Hence, the dilemma of whether sentinel node biopsy should be performed before or after preoperative chemotherapy applies only to those patients in whom there are no axillary lymph node metastases before the chemotherapy in question (cNO). Even though the question of whether to perform sentinel node biopsy before or after preoperative chemotherapy in cNO patients still remains open, a number of indicators testifying to the quality of the results obtained, tend to point in favour of sentinel node biopsy performed prior to chemotherapy. When sentinel node biopsy is performed before induction chemotherapy, the node identification rate is higher (100\% vs $95 \%$ ), and the rate of false negative results is lower $(7.4 \%$ vs $11.4 \%)$ than in the case of a post-chemotherapy biopsy.

NOWOTWORY J Oncol 2017; 67, 4: 267-269

Key words: breast cancer, sentinel node biopsy, neoadjuvant chemotherapy, false negative rate

Presently, preoperative chemotherapy is administered ever more frequently in breast cancer patients. It is administered in both non-resectable and resectable patients, i.e. those in whose case it is possible to undertake surgical treatment without the use of chemotherapy, but at the same time we know that neoadjuvant chemotherapy may further improve our treatment results. Preoperative chemotherapy is administered both in patients with baseline axillary lymph node involvement as well as in those without. Patients with lymph node involvement, reported prior to chemotherapy, may undergo complete remission, while in patients without baseline axillary lymph node involvement, metastases may sadly develop at a later stage. Hence, it is of key significance to be able to assess the condition of axillary lymph nodes accurately. When the metastases are overt, there are usually no diagnostic controversies. The doubts are there, however, when the physical examination and imaging tests indicate no lymph node metastases. We know that the sensitivity of those tests is far from ideal, and that only the sentinel node biopsy is the source of a definite confirmation of the presence of lymph node involvement or lack thereof.

A sentinel node biopsy may only be performed in patients classified as cNO. Hence, the dilemma of whether a sentinel node biopsy should be performed before or after preoperative chemotherapy applies only to those patients in whom there are no axillary lymph node metastases before the chemotherapy in question (cN0). There is no dilemma, on the other hand, when lymph node involvement is reported prior to chemotherapy. In the latter case, there are no indications for a sentinel node biopsy. It may only be performed after chemotherapy, when complete clinical remission of lymph node metastases has been observed. Therefore, $\mathrm{cN}+$ patients, prior to chemotherapy, should be excluded from the discussion that follows. Similarly, there are no indications for sentinel node biopsy following preoperative chemotherapy, when axillary lymph node invoIvement is clinically confirmed. That clinical situation will not be discussed here either. 
The question of whether to perform sentinel node biopsy before or after neoadjuvant chemotherapy in cNO patients has remained open [1]. Contemporary guidelines are not helpful in resolving the dilemma either. The NCCN recommendations indicate that biopsy may be performed before or after chemotherapy [2], which goes to say that the decision is to be taken on a case-by-case basis by the treatment team.

What speaks in favour of a sentinel node biopsy performed prior to neoadjuvant chemotherapy? First and foremost, a pre-chemotherapy biopsy makes it possible to assess the degree of disease advancement accurately enough, prior to the initiation of treatment. When there is no sentinel lymph node involvement, we may safely withdraw from post-chemotherapy axillary lymph node dissection. That approach is also reflected in the NCCN guidelines [2]. If, on the other hand, there is sentinel lymph node involvement, we may correctly qualify the patient for axillary lymph node dissection, and plan adjuvant radiotherapy appropriately. In that respect, pre-chemotherapy sentinel node biopsy is revealed as advantageous. We know that chemotherapy will result in the complete remission of axillary lymph node metastases in $20-40 \%$ of patients diagnosed with lymph node involvement prior to treatment [3]. Radiotherapy should later be offered to those patients, even if there has been complete remission of the axillary lymph node lesions. Thus, if we fail to perform sentinel node biopsy before preoperative chemotherapy, we will have no knowledge of the baseline axillary lymph node metastases, and we will be oblivious of the fact that there are indications for adjuvant axillary radiotherapy. Performing a sentinel lymph node biopsy only after neoadjuvant chemotherapy may thus result in a failure to offer a significant part of treatment to some patients.

An obvious question that arises is why not perform sentinel lymph node biopsy twice, i.e. before and after neoadjuvant chemotherapy? It could be the optimum solution. It is not, though, and study results remain incontrovertible in that respect.

The first study worth mentioning here is the SENTINA trial, a multi-centre study carried out in 103 therapeutic centres across Germany and Austria [1]. The study involved over 2200 patients. One study arm included patients with sentinel lymph node involvement, reported prior to neoadjuvant chemotherapy, who underwent chemotherapy, followed by a second sentinel node biopsy with axillary lymph node dissection. Thanks to that approach, it was possible to assess the percentage of correctly identified sentinel lymph nodes, and to determine the percentage of false negative results of the second sentinel node biopsy. As a result of the second biopsy, sentinel lymph nodes were identified in $61 \%$ of the cases only, which is an extremely low detection rate. In $52 \%$ of the cases, the results obtained were false negatives, which means that no metastases were found in the sentinel lymph node, but they were detected in other axillary lymph nodes, removed during the lymph node dissection procedure. That seems, on the other hand, an extremely high false-negative rate.

The second, more recent study was carried out by Swedish researchers [4]. Quality indicators of the second sentinel lymph node biopsy were revealed as slightly better than the ones generated in the SENTINA trial, but they are still largely unacceptable. The study, which involved 224 female patients, resulted in a $70 \%$ sentinel node detection rate, and a $25 \%$ false-negative rate.

Both of the above mentioned studies demonstrate that a repeated sentinel lymph node biopsy in patients subjected to preoperative chemotherapy is completely unreliable.

Thus, a sentinel node biopsy should only be performed once. The decision remains to be taken as to when to perform it.

As discussed above, what speaks in favour of the sentinel lymph node biopsy performed before neoadjuvant chemotherapy in cNO patients is the fact that one can assess the degree of disease advancement prior to the initiation of treatment. Another reason for performing the biopsy before rather than after preoperative chemotherapy in cNO patients involves the biopsy reliability indicators, i.e. the sentinel node detection rate and the false-negative rate.

As regards the detection rate, a sentinel node biopsy performed before preoperative chemotherapy is absolutely unrivalled, with the rate reaching $99-100 \%$. The result is based on the SENTINA study [1], involving 1022 pre-chemotherapy biopsies, and on the Swedish study [4], which involved 224 biopsies.

When sentinel node biopsy is performed after preoperative chemotherapy, its results are much less reliable. For comparison, I reviewed the results of a meta-analysis including 17 studies, which involved over 1700 breast cancer patients [5]. Detection rates ranged from $83 \%$ to $100 \%$. The authors of the meta-analysis calculated the average detection rate as $95 \%$. However, a further analysis of the individual studies included in the meta-analysis indicated that the studies that reported detection rates of $100 \%$ were very"small". In all those studies, a post-chemotherapy biopsy was only performed in 17 patients, which suggests that the reliability of those results is rather poor $[6,7]$.

The other indicator which should be examined is the false-negative rate. In the early studies on the use of sentinel node biopsy before neoadjuvant chemotherapy, false-negative rates were very low, amounting to $0 \%$ in selected trials. Such a result would be considered ideal, but is highly unlikely, and thus unreliable. The studies which generated such results involved as few as 11 or 15 biopsies, which is why no reliable conclusions could be drawn on their basis [8, 9].

On the other hand, the previously mentioned Swedish study did lead to reliable results, as it involved a group of 224 
subjects [4]. In that study, the false-negative rate was 7.4\%, which is not that small a number. It should be emphasised here that the false negative results reported also included cases of micrometastases only. Their clinical significance after chemotherapy is not yet known, which is why they were treated as significant to ensure patients' safety. What was the reason for the high rate of false negative results? Disease progression may not be excluded, which is why the authors came to the conclusion that axillary lymph node dissection may be aborted, provided there is no sentinel lymph node involvement prior to chemotherapy, and there is a good response to chemotherapy.

The false-negative rate is much higher, when the biopsy is performed after neoadjuvant chemotherapy. In the above quoted meta-analysis, whose results were published in 2013, it was calculated that the average false-negative rate for post-chemotherapy sentinel node biopsy totalled $11.4 \%$ [5]. However, in some of the studies included in the meta-analysis, the rates were as high as $14 \%, 16 \%, 17 \%$ or even $28 \%$ [5].

In summary, both indicators favour the sentinel lymph node biopsy performed before neoadjuvant chemotherapy. When sentinel node biopsy is performed prior to chemotherapy, detection rates are higher (100\% vs $95 \%)$, and false-negative rates are lower ( $7.4 \%$ vs $11.4 \%)$ than in the case of a post-chemotherapy biopsy.

As mentioned before, one argument against the sentinel node biopsy performed before neoadjuvant chemotherapy is the possibility of obtaining complete clinical remission in the lymph nodes of $20-40 \%$ of the chemotherapy patients. It goes to say that if the sentinel node biopsy is performed before neoadjuvant chemotherapy, and lymph node involvement is confirmed, standard procedure involves post-chemotherapy lymph node dissection, even though there may be complete remission of the lymph node metastases in $20-40 \%$ of the patients as a result of the chemotherapy administered. Thus, some of the patients may be overtreated. How big of a problem is it, though? Amongst the cNO patients enrolled in the SENTINA study, sentinel node biopsy performed prior to chemotherapy confirmed lymph node involvement in $40 \%$ of them. If remission occurred in $20-40 \%$ of the cases, the risk of surgical overtreatment concerned $8-16 \%$ of the patients involved. In order to establish whether that number is high or low, we have to decide what we fear more: the idea of treating people who may not need the treatment offered or failure to undertake treatment in those who may need it.

And what other arguments are there against sentinel node biopsy performed after neoadjuvant chemotherapy?
Human biology. Studies have demonstrated that following chemotherapy, lymphatic vessels undergo remodelling, with fibrotic lesions that may compromise lymphatic drainage. Advocates of post-chemotherapy biopsy indicate that lymphatic drainage is altered only in $5 \%$ of the cases [5]. But it is this $5 \%$ that matters, as this is exactly the difference between the sentinel node detection rates and the false-negative rates obtained in pre-chemotherapy and post-chemotherapy biopsies. And the difference points in favour of the biopsy performed prior to preoperative chemotherapy.

\section{Conflict of interest: none declared}

\section{Janusz Piekarski, MD, PhD}

Clinical Department of Surgical Oncology

Medical University of Łódź

Paderewskiego St. 4

93-509 Łódź, Poland

e-mail:janusz.piekarski@umed.lodz.pl

Received \& Accepted: 10 Apr 2017

Based on the presentation at the V Annual Conference of the Nowotwory Journal of Oncology, 'Oncological Debates', held in Warszawa, 7-8th April 2017

\section{References}

1. Kuehn T, Bauerfeind T, Fehm T et al. Sentinel-lymph-node biopsy in patients with breast cancer before and after neoadjuvant chemotherapy (SENTINA): a prospective, multicenter cohort study. Lancet Oncol 2013; 14: 609-618.

2. National Comprehensive Cancer Network. NCCN Guidlines:Breast cancer, Version II, 2016. www.nccn.org.

3. Kuerer HM, Sahin AA, Hunt KK et al. Incidence and impact of documented eradication of breast cancer axillary lymph node metastases before surgery in patients treated with neoadjuvant chemotherapy. Ann Surg 1999; 230: 72-78.

4. Zetterlund L, Celebioglu F, Axelsson R et al. Swedish prospective multicenter trial on the accuracy and clinical relevance of sentinel lymph node biopsy before neoadjuvant systemic therapy in breast cancer. Breast Cancer Res Treat 2017; 163: 93-101.

5. Fontein DBY, van de Water W, Mieog JSD et al. Timing of the sentinel lymph node biopsy in breast cancer patients receiving neoadjuvant therapy — recommendation for clinical guidance. EJSO 2013; 39: 417-424.

6. Tanaka $\mathrm{Y}$, Meada $\mathrm{H}$, Ogawa $\mathrm{Y}$ et al. Sentinel node biopsy in breast cancer patients treated with neoadjuvant chemotherapy. Oncol Rep 2006; 15: 927-931.

7. Reitsamer $R$, Peintinger $F$, Rettenbacher $L$ et al. Sentinel lymph node biopsy in breast cancer patients after neoadjuvant chemotherapy. J Surg Oncol 2003; 84: 63-67.

8. Schrenk P, Tausch G, Wolfl S et al. Sentinel node mapping performed before preoperative chemotherapy may avoid axillary dissection in breast cancer patients with negative or micrometastatic sentinel nodes. Am J Surg 2008; 196: 176-183.

9. Menard JP, Extra JM, Jacquemier J et al. Sentinel lymphadenectomy for the staging of clinical axillary node-negative breast cancer before neoadjuvant chemotherapy. EJSO 2009; 35: 916-920. 